\title{
Calculation of binary and ternary metallic immiscible clusters with icosahedral structures
}

\author{
Abdiravuf A. Dzhurakhalov, ${ }^{1,2, *}$ Ivailo Atanasov, ${ }^{1,3}$ and Marc Hou ${ }^{1}$ \\ ${ }^{1}$ Physique des Solides Irradiés et des Nanostructures CP234, Université Libre de Bruxelles, Bd du Triomphe, B-1050 Bruxelles, Belgium \\ ${ }^{2}$ Department of Physics (U217), University of Antwerp (CGB), Groenenborgerlaan 171, B-2020 Antwerp, Belgium \\ ${ }^{3}$ Institute of Electronics, Bulgarian Academy of Sciences, Tzarigradsko Chaussee 72, 1784 Sofia, Bulgaria \\ (Received 5 November 2007; revised manuscript received 21 January 2008; published 10 March 2008)
}

Recently, core-shell Ag-Co, $\mathrm{Ag}-\mathrm{Cu}$, and "onionlike" $\mathrm{Cu}-\mathrm{Co}$ equilibrium configurations were predicted in the case of isolated face centered cubic (fcc) bimetallic clusters, and three shell onionlike configurations were predicted in the case of ternary metallic clusters with spherical and truncated octahedral morphologies. In the present paper, immiscible binary $\mathrm{CuCo}$ and ternary $\mathrm{AgCuCo}$ clusters with icosahedral structures are studied as functions of their size and composition. Clusters studied are formed by 13, 55, 147, 309, and 561 atoms corresponding to the five smallest possible closed shell icosahedral structures. An embedded atom model potential is used to describe their cohesion. Equilibrium configurations are investigated by means of Metropolis Monte Carlo free energy minimization in the (NPT) canonical ensemble. Most simulations are achieved at 10 and $300 \mathrm{~K}$. The effect of temperature on segregation ordering is systematically investigated. Selected cases are used to identify the effect of size and composition on melting. In contrast with fcc clusters, homogeneous onionlike configurations of binary clusters are not predicted. When it is allowed by the composition, a complete outer shell is formed by $\mathrm{Cu}$ in binary $\mathrm{Cu}-\mathrm{Co}$ clusters and by $\mathrm{Ag}$ in ternary $\mathrm{Ag}-\mathrm{Cu}$-Co clusters. Depending on temperature, $\mathrm{Co}$ may precipitate into decahedral groups under the $\mathrm{Cu}$ vertices of the icosahedra in binary clusters, while the $\mathrm{Co}-\mathrm{Cu}$ configuration in ternary clusters drastically depends on the Ag coating. Despite the multicomponent character of the clusters and the immiscibility of the species forming them, for most compositions and sizes, equilibrium structures remain close to perfectly icosahedral at $10 \mathrm{~K}$ as well as at $300 \mathrm{~K}$.

DOI: 10.1103/PhysRevB.77.115415 PACS number(s): 61.46.Bc, 61.43.Bn, 61.50.Ah, 81.30.Mh

\section{INTRODUCTION}

Metallic nanoparticles trigger fundamental interest for their confinement properties as well as in view of applications in nanotechnologies. Indeed, the possibility of tuning their size, morphology, structure, and composition opens routes to original properties and to their monitoring in view of their use in nanoscale devices.

When the cluster size is sufficiently confined, fivefold symmetrical structures and morphologies turn out to be better stable than cubic, even when they are made of more than one element. For instance, the configurational energies of icosahedral homotops of $\mathrm{Ni}_{n} \mathrm{Al}_{p}$ clusters with $n+p=12,13$, and 14 were systematically studied ${ }^{1}$ on the basis of both experimental measurements and theoretical considerations. Reviews emphasizing the efficiency of genetic algorithms for geometry optimization of small clusters demonstrate the stability of the fivefold topologies. ${ }^{2,3}$ A general review of computational methods developed in order to study such cluster structures and properties is presented in Ref. 4 while, not much later, another review of experimental characterization of clusters crystallography was published. ${ }^{5}$ A recent review reports about the major trends in computational and experimental studies of bimetallic clusters. ${ }^{6}$

Because of their small size, the experimental characterization of fivefold symmetrical clusters may be difficult, in particular, when they are made of more than one element. A number of reports are published, however. A study of the influence of individual metals on the stability of mixed clusters was already reported in Ref. 7. Similar studies using mass spectrometry and laser fragmentation are now well known. ${ }^{8-12} \mathrm{~A}$ variety of structures and mor- phologies of Au-Cu clusters was identified, ${ }^{13-17}$ as well as in other bimetallic systems. ${ }^{18-28}$ On the theoretical side, fivefold symmetries were predicted using the density functional theory (DFT) in both elemental and binary small clusters ${ }^{29-31}$ sometimes in combination with semiempirical techniques. ${ }^{32-35}$

Global optimization techniques require a huge amount of iterations and were used therefore with semiempirical potentials, ${ }^{36-40}$ with the generic Lennard-Jones potential, ${ }^{41}$ or using semiempirical potentials for a first optimization and DFT for a second optimization. ${ }^{42-46}$ Such methods allow addressing a large amount of case studies involving clusters with larger sizes than full DFT computations. However, the needed computational effort nowadays limits the investigated cluster sizes in the range of 100 atoms. In this range, fivefold symmetry is still dominant, and a significant insight is now available about segregation and its relationship with the stability of homotops.

As larger clusters are concerned, molecular dynamics (MD) and Metropolis Monte Carlo (MMC) with semiempirical potentials are still the best efficient approaches, sometimes coupled with global optimization. MD and MMC allow studying kinetic and thermodynamic properties of clusters with up to many thousands of atoms realistically and the literature is abundant. Regarding bimetallic clusters, ordered, disordered, and segregated fivefold symmetric structures are predicted. For instance, in Au-Cu clusters, ${ }^{47}$ as well as in other compounds, ${ }^{48} \mathrm{MD}$ was systematically used to identify the conditions at which icosahedral, decahedral, or cuboctahedral morphologies take place. The role of temperature was investigated ${ }^{49}$ and the factors governing cluster stability were discussed. ${ }^{50-58}$ 
Only limited literature about clusters formed by more than two metals and having a fivefold symmetry is presently available. However, icosahedral clusters were identified experimentally by extended x-ray-absorption fine structure (EXAFS) in $\mathrm{Zr}_{70} \mathrm{Cu}_{29} \mathrm{Pd}_{1}$ metallic glasses. ${ }^{59}$ The thermal stability of Al-Pd-Mn icosahedral clusters formed by laser vaporization of quasicrystals was studied. ${ }^{60}$ Theoretically, a thermodynamic approach was used to study surface segregation in ternary metal clusters with icosahedral and cuboctahedral morphologies. ${ }^{61-64}$

In a previous study, the equilibrium properties of metallic fcc clusters with truncated octahedral morphologies and made of two and three different immiscible elements were investigated in detail at the atomic scale. ${ }^{65}$ Metropolis Monte Carlo simulations in the canonical (NPT) ensemble were used therefore. Typical sizes of such fcc clusters experimentally synthesized range from a few hundred to several thousands of atoms. In smaller clusters that are also currently synthesized experimentally, fivefold symmetry is privileged and clusters with suitable numbers of atoms form perfect icosahedral clusters. Neither MMC nor MD techniques are efficient in predicting optimal cluster morphologies and cluster sizes at which the icosahedral to cubic transition occurs, and this transition may depend on the environment.

In the present study, we acknowledge the possibility of both structures and we address the question to which extend the properties identified in fcc clusters also pertain to the icosahedral structure. The three elements considered in Ref. 65, Ag, $\mathrm{Cu}$ and $\mathrm{Co}$, are also studied here. An embedded atom model (EAM) is used in order to describe the cohesion of the compounds. ${ }^{66-68}$ As the elemental solids are concerned, the equilibrium potential is conveniently assessed, for instance, by comparing experimental and predicted thermal expansion coefficients and mean square thermal vibration amplitudes. In the case of compounds, the assessment is more delicate since $\mathrm{Ag}, \mathrm{Cu}$, and $\mathrm{Co}$ form no alloy in the bulk for which similar properties could be measured at equilibrium. Using EXAFS and Mössbauer spectroscopy data, it was, however, possible to assess the AgCo potential. ${ }^{69}$ To our knowledge, similar experimental data are not available for the $\mathrm{AgCu}$ and the $\mathrm{CoCu}$ systems, and it is assumed that the same EAM still reasonably holds. This was supported by the computation of the heats of solution of the three $\mathrm{Ag}-\mathrm{Co}, \mathrm{Ag}-\mathrm{Cu}$, and $\mathrm{Cu}-\mathrm{Co}$ systems versus composition and by the comparison with experimental and theoretical studies of nonequilibrium alloys. ${ }^{70-76}$ In the compound clusters, all heats of solution were found positive, demonstrating that, like in the bulk, Ag, $\mathrm{Co}$, and $\mathrm{Cu}$ are immiscible in nanoclusters.

$\operatorname{Ag}_{n-x} \mathrm{Co}_{x}$ fcc clusters with $200<n<3000$ were already examined in great detail elsewhere. ${ }^{57}$ It was found that, in the solid state, the atomic configurations of both species result from a balance between lattice distortion and binding energy, and similar results were found with $\mathrm{Ag}_{n-x} \mathrm{Cu}_{x}$ clusters. When $x \leqslant 10$, it costs less energy to locate the $\mathrm{Co}(\mathrm{Cu})$ atoms in small groups just beneath the surface and displacing the Ag surface atoms therefore than to regroup all $\mathrm{Co}(\mathrm{Cu})$ atoms at the center. At large $x$ values, a core-shell structure with Co $(\mathrm{Cu})$ in the core is privileged. The situation is significantly different for fcc $\mathrm{Cu}_{n-x} \mathrm{Co}_{x}$ which heat of solution is smaller. In this case, at small $x$, Co is distributed uniformly beneath the $\mathrm{Cu}$ surface layer and a full Co subsurface layer could be predicted, giving rise to an onion structure. This onion structure disappears if the cluster is coated with an additional surface layer of Ag. ${ }^{65}$

In what follows, the same Metropolis Monte Carlo method as in our previous work is used and we focus on small $\mathrm{CuCo}$ and $\mathrm{AgCuCo}$ icosahedral clusters, and their equilibrium configurations are systematically studied as functions of size and composition.

\section{CuCo CLUSTERS WITH ICOSAHEDRAL STRUCTURES}

The geometric magic numbers associated with clustersknown as Mackay icosahedra-composed by $\mathrm{K}$ complete icosahedral shells, with multiple fivefold symmetry axes, are given by ${ }^{77}$

$$
N(K)=\frac{1}{3}\left(10 K^{3}+15 K^{2}+11 K+3\right) .
$$

The first five clusters in this series have, respectively, 13 $(K=1), 55(K=2), 147(K=3), 309(K=4)$, and $561(K=5)$ atoms. The number of atoms in the first shell is 12 , in the second shell 42, in the third shell 92, 162 in the fourth, and 252 in the fifth. In the 13-atom cluster, all atoms (except the central one) occupy equivalent sites. The 55-atom cluster in this series consists of a core-which is just the 13-atom icosahedron-12 atoms at the vertices and 30 atoms at the center of each of the 30 edges of the icosahedron. The 147atom cluster has an additional shell where one atom occupies the center of each facet. No new site types are generated in larger icosahedra. The binary $\mathrm{Cu}_{n-m} \mathrm{Co}_{m}$ clusters with $n=13$, $55,147,309,561$ and $0 \leqslant m \leqslant n$ are considered in this section. We systematically study clusters at low temperature $(10 \mathrm{~K})$, comparing their configurations with those at $300 \mathrm{~K}$ in order to investigate sensitivity of configurations to temperature. The effect of composition on the melting temperature of binary clusters is only briefly commented.

In several instances, in particular, in small ternary clusters, the differences between zero temperature configuration energies of several isomers were such small that, because of the magnitude of thermal fluctuations, it was not possible to identify the best stable at $300 \mathrm{~K}$ despite the large number of MMC steps used $\left(5 \times 10^{6}\right.$ moves per atom and $50000-$ 500000 exchange trials per atom pairs). In such cases, the identification of global minimum Helmholtz free energy configurations is not warranted. However, the configuration differences between such isomers, when identified, were small.

For convenience, the same convention is used in cluster snapshots shown in the figures: Dark (pink in color version) spheres represent $\mathrm{Cu}$ atoms, midgray (blue in color version) spheres represent Ag atoms, and light gray spheres represent Co atoms.

\section{A. 13-atom clusters}

In Figs. 1(a)-1(f), the equilibrium configurations of $\mathrm{Cu}_{13-m} \mathrm{Co}_{m}$ clusters are shown for several values of $m$ at a temperature of $10 \mathrm{~K}$. It is seen that the central position is always occupied by $\mathrm{Co}$. With increasing $m$, the $\mathrm{Cu}$ atoms are 

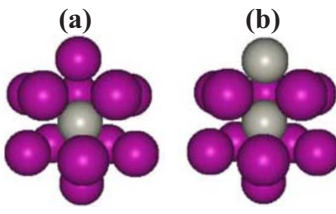

(e)

(d)
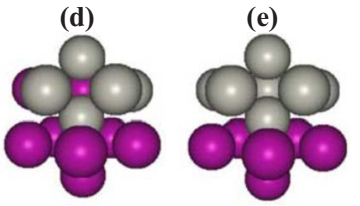

(h)
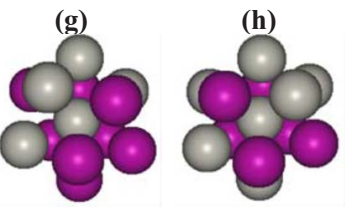

(c)

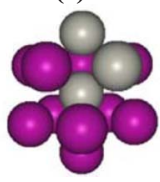

(f)

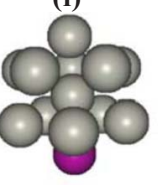

\section{(1)}

FIG. 1. (Color online) Icosahedral $\mathrm{Cu}_{13-m} \mathrm{Co}_{m}$ clusters at $T$ $=10 \mathrm{~K}$ with (a) $m=1$, (b) $m=2$, (c) $m=3$, (d) $m=5$, (e) $m=7$, (f) $m=12$, and at $T=300 \mathrm{~K}$ with (g) $m=5$ and (h) $m=7$.

replaced by $\mathrm{Co}$ atoms forming a compact first neighbor group. Despite of the breakdown of symmetry, the final configurations are perfect icosahedra.

The icosahedral structure of the six homotops displayed in Fig. 1 is maintained at $300 \mathrm{~K}$. For $m \leqslant 2$ and $m=12$, the configurations are unchanged as well. When the numbers of $\mathrm{Co}$ and $\mathrm{Cu}$ atoms are comparable, the increase of temperature tends to reduce the segregation efficiency and the distributions of the species in the clusters get more homogeneous, as seen by comparing the Figs. 1(d) and 1(e) with $1(\mathrm{~g})$ and $1(\mathrm{~h})$.

\section{B. 55-atom cluster}

Figure 2 shows the final configurations of $\mathrm{Cu}_{55-m} \mathrm{Co}_{m}$ clusters for some values of $m$ at $10 \mathrm{~K}$. In this case, when 0 $<m<10$, the Co atoms are located in small groups just beneath the surface $(K=1)$ and the central atom is $\mathrm{Cu}$ [Figs. 2(a) and 2(b)]. This pertains to both 10 and $300 \mathrm{~K}$. At $300 \mathrm{~K}$, however, the six Co atom configuration is not as compact as shown in Fig. 2(b), but their positions keep confined in the

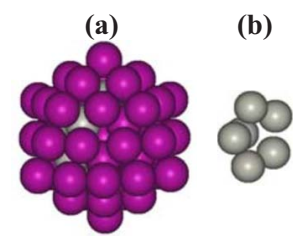

(d)

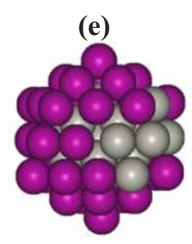

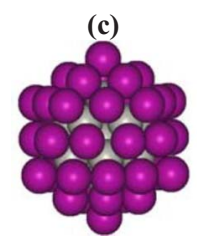

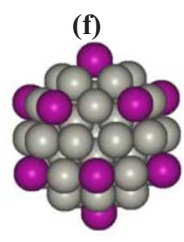

FIG. 2. (Color online) Icosahedral $\mathrm{Cu}_{55-m} \mathrm{Co}_{m}$ clusters. (a) $m$ $=6$, (b) the $\mathrm{Co}_{6}$ group in (a), (c) $m=13$, (d) the $\mathrm{Co}_{13}$ group in (c), (e) $m=19$, and (f) $m=43$. The temperature is $T=10 \mathrm{~K}$.

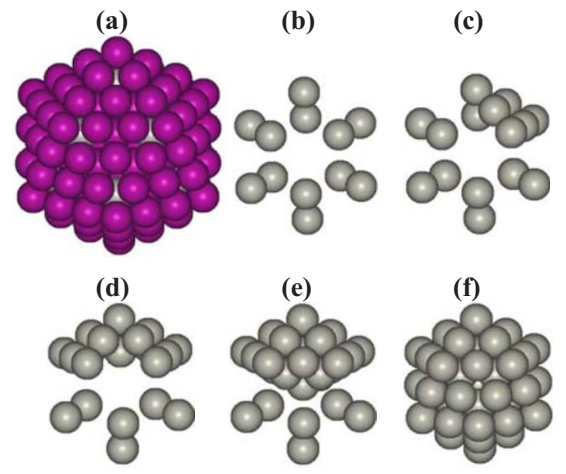

FIG. 3. (Color online) Icosahedral $\mathrm{Cu}_{147-m} \mathrm{Co}_{m}$ clusters. (a) $m$ $=12$, (b) same as (a), but only Co atoms are shown, (c) $m=15$, (d) $m=22$, (e) $m=30$, and (f) $m=55$. In (c)-(f), only Co atoms are shown. The temperature is $T=10 \mathrm{~K}$.

$K=1$ cluster shell. Further increasing $m$ results in filling the core of the cluster with $\mathrm{Co}$, and when $m=13$, the Co forms the 13-atom inner icosahedron, covered by the $42 \mathrm{Cu}$ atoms closing the outer shell [Figs. 2(c) and 2(d)]. This close shell structure remains stable for the higher temperature of $300 \mathrm{~K}$. Notice that in principle, at $m=12$, Co could form a closed $K=1$ shell. In fact, one $K=1$ site is occupied by $\mathrm{Cu}$ and the central site by one Co atom. The $K=1$ shell is thus never closed by $\mathrm{Co}$ atoms. When $m>13, \mathrm{Cu}$ atoms of the outer shell are consequently replaced by $\mathrm{Co}$ atoms. The $\mathrm{Cu}$ atoms forming the edges are replaced first and then those at the vertices [Fig. 2(e)]. At $m=43$ and $10 \mathrm{~K}$, the $12 \mathrm{Cu}$ atoms sit at the 12 vertices of the icosahedron [Fig. 2(f)]. At $300 \mathrm{~K}$, this symmetrical $\mathrm{Cu}$ arrangement is partially disordered.

\section{147-atom clusters}

The case of $\mathrm{Cu}_{147-m} \mathrm{Co}_{m}$ clusters differs from the previous ones. Configurations of Co atoms for selected $m$ are shown in Fig. 3 at $10 \mathrm{~K}$. When $m \leqslant 12$, the Co atoms are homogeneously distributed in the subsurface shell $(K=2)$ and occupy the vertices. This configuration is symmetrical to that of 55atom cluster containing $12 \mathrm{Cu}$ atoms instead of $12 \mathrm{Co}$ in the $K=2$ shell. The effect of temperature is similar as well, namely, to delocalize some Co atoms to edges in the same shell. Such a homogeneous subsurface configuration is similar to that found in fcc clusters; however, the situation changes for larger $m$ values before a full shell of Co can be formed. Indeed, at $10 \mathrm{~K}$, increasing $m$ above 12 results in filling one facet of the subsurface shell by Co atoms at first and then the adjacent ones (at $12<m<22$ ) [Figs. 3(c) and $3(\mathrm{~d})$ ]. The $K=1$ site at their intersection is occupied by a $\mathrm{Co}$ atom too, forming a seven-atom decahedron with its $K=2$ first neighbors. Temperature partially inhibits this faceting. At $300 \mathrm{~K}$, edge positions are privileged and no decahedron is formed. Co mainly remains in the $K=2$ shell and, in some configurations, Co atoms appear at the cluster surface. When $m$ is still increased further [Fig. 3(e)], the additional Co atoms do not close the shell. Indeed, at $10 \mathrm{~K}$, they form a decahedral object based on the filled adjacent facets. From there on, the growth of the Co cluster is three dimensional and completes the core at $m=55$ [Fig. 3(f)]. This completion 


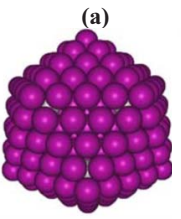

(d)

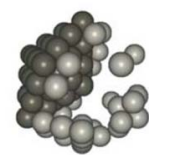

(b)

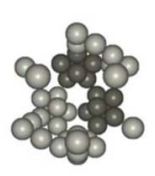

(e)

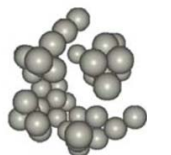

(c)

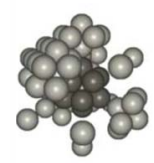

(f)

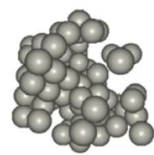

FIG. 4. (Color online) Icosahedral $\mathrm{Cu}_{309-m} \mathrm{Co}_{m}$ clusters. Lowest energy configurations identified at a temperature $T=10 \mathrm{~K}$ are shown in (a)-(d) with (a) $m=42$, only $\mathrm{Cu}$ atoms are seen, (b) $m$ $=42$, only Co atoms are displayed and decahedra formed by seven atoms are shown in dark, (c) $m=72$, an isomer configuration only slightly less favorable energetically than the decahedral pattern shown in (b), and (d) $m=84$, Co is regrouped in a large decahedron shown in dark. Lowest energy configurations identified at a temperature $T=300 \mathrm{~K}$ are shown in (e) and (f) with (e) $m=42$ and (f) $m=72$.

is also observed at $300 \mathrm{~K}$. However, no similar decahedron is formed as shown for $m=30$ in Fig. 3(e). Instead, Co is predominantly filling the $K=2$ shell before the $K=1$ shell. Hence, as it was already found in the 55-atom clusters, temperature favors the onionlike structure at the expense of decahedron formation; however, a close shell onion layering is not achieved.

\section{309-atom cluster}

For the sake of clarity, the discussion of configurations at 10 and $300 \mathrm{~K}$ is distinguished in this section and we start with the case of low temperature.

Similarly to the case of $\mathrm{Cu}_{147-m} \mathrm{Co}_{m}$ clusters, $\mathrm{Co}$ atoms occupy positions beyond the surface layer insofar $\mathrm{Cu}$ is not yet fully replaced by $\mathrm{Co}$ in the inner shells. The privileged sequence as $m$ increases from zero is shown in Fig. 4. First, the 12 vertices of the subsurface shell $[K=3$ in Eq. (1)] are occupied by $\mathrm{Co}$. Next, $\mathrm{Cu}$ is replaced by $\mathrm{Co}$ at the positions of the edges in the same shell, either intersecting at a vertex or joining two neighboring vertices. For $30<m<70$, a new configuration building block of Co atoms occurs. Decahedra with seven Co atoms are formed, occupying a vertex of the $K=3$ shell, surrounded by five Co nearest neighbors in the same shell and one Co nearest neighbor at the vertex of the underlying $K=2$ shell [Fig. 4(b)]. Isomeric Co configurations were found with a slightly higher configuration energy (no more than $0.5 \mathrm{meV} /$ atom), where Co tetrahedra, based on one triangular $K=3$ facet, are adjacent to each other. Two such tetrahedra may be encountered when $m>55$. The central core of 13 atoms also appears to be a stable configuration in combination with the tetrahedra [Fig. 4(c)]. With 12 small decahedra similar to those shown in Fig. 4(b), a regular pattern of 84 Co atoms would be formed. As discussed below, this occurs in larger clusters, but not in the present one, as a consequence of the limited cluster size. Instead, for $m>70$,

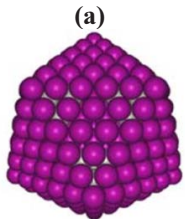

(d)

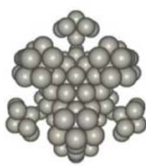

(b)

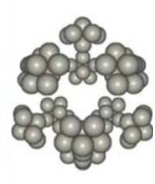

(e)

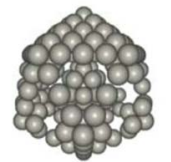

(c)

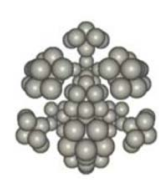

(f)

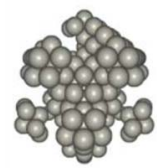

FIG. 5. (Color online) Icosahedral $\mathrm{Cu}_{561-m} \mathrm{Co}_{m}$ clusters. (a) $m$ $=84$ (Co inside), (b) $m=84$, (c) $m=97$, (d) $m=139$, a configuration with a large core and a subsurface decahedral pattern, (e) $m=139$, a configuration with a small core, and (f) $m=162$.

another configuration becomes energetically favored. It is a big decahedron formed by five adjacent tetrahedra as shown in Fig. 4(d). Similarly to the case of the 147-atom clusters, the pattern formed by cobalt below the surface layer of copper is thus not homogeneous. It initiates at few subsurface sites only and grows from these nuclei as $m$ is increased until a single Co decahedron is formed. When $m>147$, Co occupancy of surface sites starts. Surface Co atoms tend to be grouped together, forming nearest neighbor islands. No preferential location was observed for these surface islands. In general-though not systematically - the coordination of cobalt atoms is maximized and they avoid the $K=4$ vertex positions accordingly. We now turn to the configurations of the same homotops at $300 \mathrm{~K}$.

Similarly to smaller clusters, with $m \leqslant 12$, all Co atoms are located in the subsurface shell ( $K=3$ in this case), but not systematically at vertices, and edges for the case when $m$ are increased above 12. The formation of seven-atom decahedra when $m<70$ is not as systematic as at $10 \mathrm{~K}$ [Fig. 4(b)], although, as shown in Fig. 4(e), some decahedra are partially formed. No regular pattern is observed, and some Co atoms could be found, located randomly in the volume of the cluster.

In contrast with the low temperature case, at larger $m$ values, neither the central group nor a large decahedron is completely formed at $T=300 \mathrm{~K}$, and, instead, like in smaller clusters, the trend is that higher temperature favors an onion structure, which is never close shell. The consequence of temperature is also the emergence of some Co atoms at the cluster surface.

\section{E. 561-atom cluster}

We start with the discussion of low temperature homotops. Selected Co configurations predicted at $10 \mathrm{~K}$ are depicted in Fig. 5. The way cobalt atoms arrange to achieve the lowest energy configuration in $\mathrm{CuCo}$ icosahedral clusters of size 561 does not differ much from the 309-atom cluster, but the larger size allows the different patterns to be better distinguishable. Again, for $m \leqslant 12$, cobalt atoms occupy the 12 vertices of the subsurface layer. Further increasing $m$ results in forming seven-atom decahedra beneath the $K=5$ vertices 
[Fig. 5(b)]. The growth of such decahedra always nucleates from a group of three or more atoms. Co dimers are never observed at a vertex. Note that when $70<m<97$, two Co configurations are observed: either with a central icosahedral core of 13 atoms or without it, with a slightly lower configuration energy attributed to the matter. For instance, the energy difference between these two configurations for $m=72$ is no more than $2 \times 10^{-4} \mathrm{eV} /$ atom, yet distinguishable with respect to the typical standard deviation of the configuration energy, $5 \times 10^{-5} \mathrm{eV} /$ atom, during the MMC simulations. Another peculiarity of the cobalt atoms configurations is more clearly evident than in the 309-atom cluster as well. For $m$ $=97$, the minimal energy configuration consists in 12 decahedra and a central icosahedral core of 13 atoms [Fig. 5(c)]. Thanks to the larger cluster size, they are not entangled as in the smaller cluster. One thus retrieves an onion structure as previously predicted in fcc clusters, however, made of small groups rather than forming a uniform monolayer. When $m$ $\geqslant 97$, the configuration with a central icosahedral core of 55 atoms also is possible [Fig. 5(d)]. Its configuration energy is slightly higher for $m<140$ and slightly lower for $m \geqslant 140$. Two isomeric Co configurations for $m=139$ are depicted in Figs. 5(d) and 5(e) with a central icosahedral core of 55 atoms and with a central icosahedral core of 13 atoms, respectively. It is seen that after forming the 12 vertex decahedra, cobalt atoms preferentially take positions in the $K=4$ shell, starting from the icosahedral edges, then facets, and then $K<4$ shells [see also Fig. 5(f)]. In all the cases, the 12 vertex decahedra persist, which demonstrates their particularly high stability.

We now comment on the differences in configurations at 10 and $300 \mathrm{~K}$. At $300 \mathrm{~K}$, for $m=84$, the pattern of 12 decahedra is largely vanished. Co tends to fill subsurface layers $(K=4)$, preferentially along edges, and a few atoms are scattered at surface sites as well as in inner shells. The icosahedral 13 Co core observed at $10 \mathrm{~K}$ for $m>97$ vanishes at $300 \mathrm{~K}$ as well as the seven-atom subsurface decahedra. The onion layering is not homogeneous, however, and substantial thermal disorder takes place. The poor uniformity of the onion structure at $300 \mathrm{~K}$ is not improved by higher temperature. Configurations were calculated at $600 \mathrm{~K}$, displaying an increased disorder and an increased emergence of Co atoms at the cluster surface, but no enhancement of the onion layering.

\section{HIGH TEMPERATURE EFFECTS}

So far, the role of entropy is mainly estimated at low and up to room temperatures. We now turn to the discussion of high temperature thermal disordering. In order to emphasize the effect of temperature, the same free energy minimizations as in the previous section are performed in a temperature range up to $1500 \mathrm{~K}$. At moderate temperature, clusters keep their icosahedral structure. At high temperature, site exchanges are found possible resulting in some disorder. The transition between the low and the high temperature regimes is documented in Ref. 78. Above $700 \mathrm{~K}$, depending on composition and size, the icosahedral structure vanishes. Caloric curves were established by means of MD for several clusters,

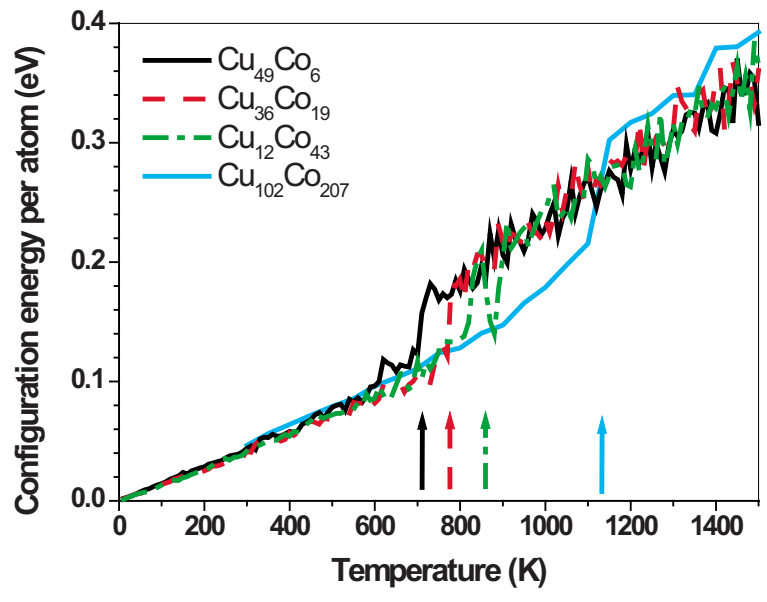

FIG. 6. (Color online) Caloric curves for $\mathrm{Cu}_{55-m} \mathrm{Co}_{m}$ clusters with $m=6,19,43$ and $\mathrm{Cu}_{102} \mathrm{Co}_{207}$. The curves are shifted to the same reference energy at $0 \mathrm{~K}$ for the sake of comparison. The true configuration energies at $T=0 \mathrm{~K}$ are $-2.9825,-3.2047,-3.5692$, and $-3.7609 \mathrm{eV}$ for clusters $\mathrm{Cu}_{49} \mathrm{Co}_{6}, \mathrm{Cu}_{36} \mathrm{Co}_{19}, \mathrm{Cu}_{12} \mathrm{Co}_{43}$, and $\mathrm{Cu}_{102} \mathrm{Co}_{207}$, respectively. The estimated melting temperatures are shown by vertical arrows.

indicating that this is a melting transition. Examples are given in Fig. 6. Because of the occurrence of jumps between the solid and the liquid states on the long term, a melting temperature could not be identified accurately. The results in Fig. 6, however, qualitatively indicate that the transition temperature increases with the Co content and with size. Even in the liquid phase, however, Co does not dissolve and, at low concentration, it remains grouped beneath the surface.

\section{TERNARY ICOSAHEDRAL CLUSTERS}

We now turn to the effect of adding Ag to the clusters studied in the previous sections, thus forming a $\mathrm{Ag}_{n-m-k} \mathrm{Cu}_{m} \mathrm{Co}_{k}$ ternary system. This leads to profound rearrangement of $\mathrm{Co}$ and $\mathrm{Cu}$, leaving the icosahedral structures stable.

Among the huge possible combinations of $n, m$, and $k$, the ternary $\mathrm{Ag}_{13-m-k} \mathrm{Cu}_{m} \mathrm{Co}_{k}$ clusters with $0 \leqslant(m+k) \leqslant 13$ and the $\mathrm{Ag}_{55} \mathrm{Cu}_{12} \mathrm{Co}_{1}, \quad \mathrm{Ag}_{147} \mathrm{Cu}_{12} \mathrm{Co}_{1}, \quad \mathrm{Ag}_{147} \mathrm{Cu}_{54} \mathrm{Co}_{1}, \quad$ and $\mathrm{Ag}_{147} \mathrm{Cu}_{42} \mathrm{Co}_{13}$ clusters are selected. Next, we select configurations of $\mathrm{Ag}_{n-m-k} \mathrm{Cu}_{m} \mathrm{Co}_{k}$ clusters with $n=55,147$ and 0 $\leqslant(m+k) \leqslant n$, with $m$ and $n$ such that the number of each species corresponds to the number of sites available in one icosahedral shell. This way, it is possible to discuss the necessary conditions for each shell to have a single elemental composition.

\section{A. 13-atom clusters}

In this case, only minor temperature effects are detected at $300 \mathrm{~K}$. The effect of increasing the $\mathrm{Cu}$ concentration in 13atom $\mathrm{Ag}_{13-m-k} \mathrm{Cu}_{m} \mathrm{Co}_{k}$ clusters with $k=1$ is shown in the first row of Fig. 7 and with $k=2$ in the second row. Configurations with fixed $m=1$ are shown in the third. In all cases, the central site is occupied by a Co atom and, as soon as $k=7$ (third row in Fig. 7), a perfect Co decahedron is formed, 


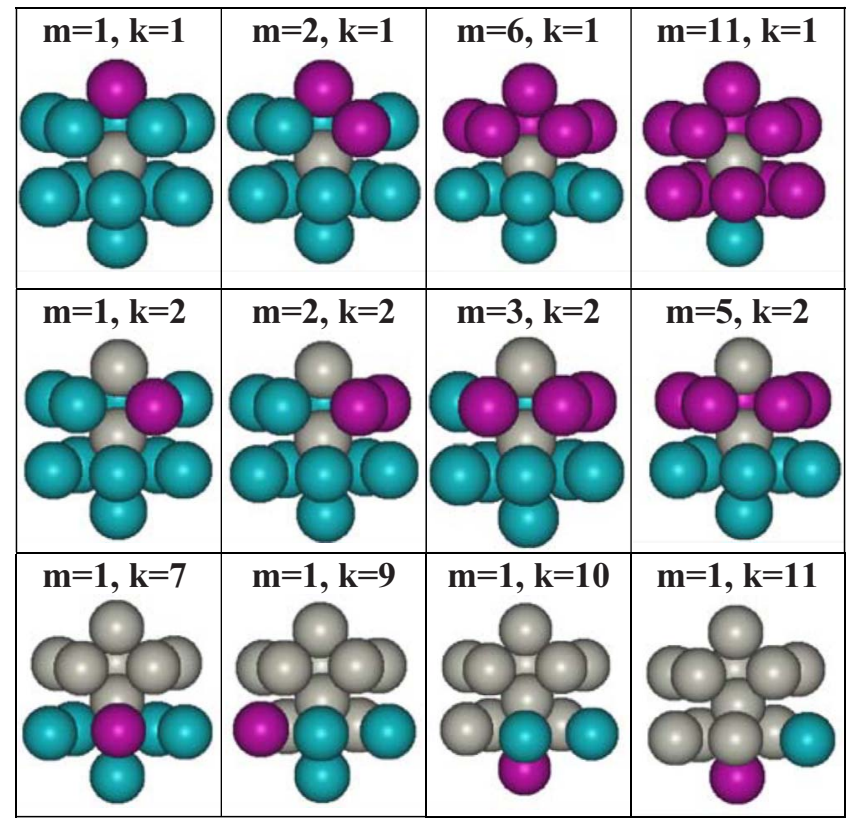

FIG. 7. (Color online) 13-atom $\mathrm{Ag}_{13-m-k} \mathrm{Cu}_{m} \mathrm{Co}_{k}$ clusters configurations with different $m$ and $k$. The temperature is $T=10 \mathrm{~K}$.

which is decorated by the additional Co atoms when $k>7$. Hence, in all cases, Co makes first neighbor bonds with other Co atoms whenever possible. The first and second rows in Fig. 7 illustrate that $\mathrm{Cu}$ and $\mathrm{Ag}$ do not mix either. Phase separation at 10 and $300 \mathrm{~K}$ is thus clear, even in such small clusters. In addition, as a consequence of segregation between the species, minority elements are grouped together. This is depicted in Fig. 7. In the first two rows of the figure, $\mathrm{Cu}$ is shown to regroup with the Co group, while in the third row, it is seen to regroup with Ag. The effect is mostly pronounced at $10 \mathrm{~K}$. In the best stable configurations that are found, $\mathrm{Cu}$ not only regroups with the minority element, but also the $\mathrm{Cu}-\mathrm{Cu}$ coordination is maximized.

\section{B. 55- and 147-atom clusters}

For the 55-atom cluster, the case of $m=12$ and $n-m-k$ $=42$ (with $k=1$ ) is studied. $m$ corresponds to the number of atoms in the first shell $(K=1)$ and $n-m-k$ to the number of atoms in the second shell $(K=2)$. It is seen in Fig. 8 that in the case of the $\mathrm{Ag}_{42} \mathrm{Cu}_{12} \mathrm{Co}_{1}$ cluster at $10 \mathrm{~K}$, the only Co atom is in the centre surrounded by 12 atoms of $\mathrm{Cu}$, and this $\mathrm{CuCo}$ core is covered by 42 atoms of $\mathrm{Ag}$ [Figs. 8(a) and $8(\mathrm{~b})]$. This feature remains unchanged at $300 \mathrm{~K}$. The $\mathrm{Cu}_{12} \mathrm{Co}$ icosahedral core is preserved in the 147-atom cluster whenever the shell at $K=3$ is occupied by $\mathrm{Cu}$ or $\mathrm{Ag}$. When $k$ $=13$, the central icosahedron is occupied by Co. At $300 \mathrm{~K}$, minor interlayer mixing is observed.

In Fig. 9, the equilibrium configurations of binary 55atom $\mathrm{Cu}_{55-m} \mathrm{Co}_{m}$ clusters [(a)-(e)] are compared for selected values of $m$ with the ternary 147-atom $\mathrm{Ag}_{92} \mathrm{Cu}_{55-m} \mathrm{Co}_{m}$ clusters $[(\mathrm{f})-(\mathrm{j})]$ having the same $\mathrm{CuCo}$ core at $10 \mathrm{~K}$. This $\mathrm{Ag}$ coating modifies the surface energy of the $\mathrm{Cu}_{55-m} \mathrm{Co}_{m}$ inner cluster with the consequence that the subsurface Co location becomes energetically unfavorable. In addition to the feeding
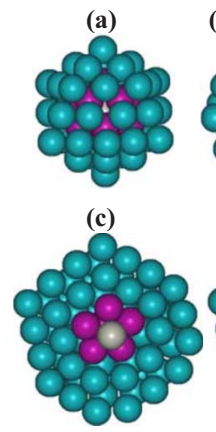

(b)

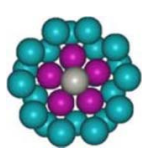

(d)

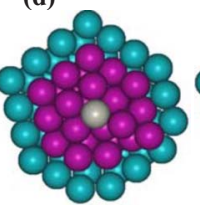

(e)

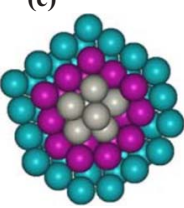

FIG. 8. (Color online) 55- and 147-atom clusters at $10 \mathrm{~K}$. (a) $\mathrm{Ag}_{42} \mathrm{Cu}_{12} \mathrm{Co}_{1}$, (b) its cross section, (c) the cross section of the $\mathrm{Ag}_{134} \mathrm{Cu}_{12} \mathrm{Co}_{1}$ cluster, (d) of the $\mathrm{Ag}_{92} \mathrm{Cu}_{54} \mathrm{Co}_{1}$ cluster, and (e) of the $\mathrm{Ag}_{92} \mathrm{Cu}_{42} \mathrm{Co}_{13}$ cluster.

of Co to the center by the Ag coating, Co forms more compact groups than without coating. The copper distribution at the surface of the $\mathrm{Cu}_{55-m} \mathrm{Co}_{m}$ clusters also strongly differs from its distribution at the interface with $\mathrm{Ag}$ in the $\mathrm{Ag}_{92} \mathrm{Cu}_{55-m} \mathrm{Co}_{m}$ ternary cluster. Figure 9 shows, for instance, that the $12 \mathrm{Cu}$ atoms in Fig. 2(f) [reproduced in Fig. 9(e)] do not occupy vertices anymore but regroup along an edge and in a facet of the same shell in the $\mathrm{Ag}_{92} \mathrm{Cu}_{12} \mathrm{Co}_{43}$ cluster [Fig. 9(j)]. The effect of the Ag coating is the same at both temperatures investigated. Little disorder is found at $300 \mathrm{~K}$ as compared to the $10 \mathrm{~K}$ configurations shown in Fig. 9, involving some atomic mixing at the surface.

\section{DISCUSSION}

The interplay between cluster size, morphology, structure and composition, surface and interface excess energies, binding energies between species, thermal disorder, and thermal vibration effects makes it impossible to predict equilibrium thermodynamic states of clusters on intuitive grounds. Guidelines may be found, however, based on the comparison over a large set of data as it was shown, for instance, in Ref. 1 in the case of 12- to 14-atom bimetallic clusters. The pur-
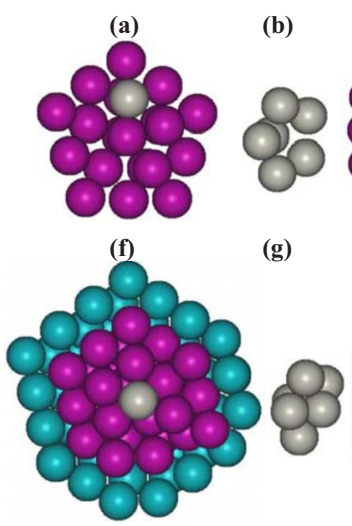

(b)

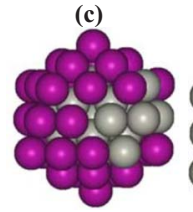

(h)

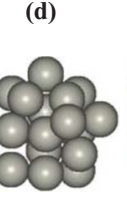

(i)

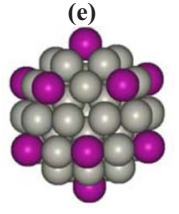

(j)
FIG. 9. (Color online) The 55-atom $\mathrm{Cu}_{55-m} \mathrm{Co}_{m}$ clusters. (a) $m$ $=1$, (b) $m=6$ (Co core), (c) $m=19$, (d) $m=19$ (Co core), (e) $m$ $=43$, and (f) the 147-atom $\mathrm{Ag}_{92} \mathrm{Cu}_{55-m} \mathrm{Co}_{m}$ clusters for the same values of $m$ (f)-(j). The temperature is $T=10 \mathrm{~K}$. 
pose of this discussion is to emphasize those that may emerge from the present work.

First, we point out that, because of its stochastic nature at the atomic scale, the MMC method used here is inefficient in predicting structural and morphological transitions involving correlated motions of large amplitude. Molecular dynamics is not better successful either because of the magnitude of the energy barriers involved in these transitions. Moreover, free energies associated with different states cannot be calculated accurately without thermodynamic integration, requiring computational effort at least 1 order of magnitude larger than the one for free energy minimization. For these reasons, energy balances are often made at $0 \mathrm{~K}$ with the hope that they pertain to higher temperatures.

Regarding the cluster structure, an MD algorithm was used in the context of the present work to perform quasistatic estimates of elemental $\mathrm{Ag}, \mathrm{Cu}$, and $\mathrm{Co}$ configuration energies per atom at $0 \mathrm{~K}$ for fcc and icosahedral clusters with sizes ranging from $N=50$ to $N=5000$ atoms. The excess energies were estimated using the fcc bulk configuration energies per atom as a reference. In this range, the $0 \mathrm{~K}$ excess energy per atom scales with $N^{-1 / 3}$ for both structures as expected, and the excess energy difference between the two structures obeys the same scaling law. We find that the icosahedral structure is energetically more favorable at $0 \mathrm{~K}$; however, the excess energy differences are typically smaller than $0.01 \mathrm{eV} /$ atom ( $5 \mathrm{meV}$ in the case of 500 atom clusters) and thus of the order of typical thermal energies. Hence, a crossover in the size dependencies of Helmholtz free energy is possible at some nonzero temperature, although none is found for $0 \mathrm{~K}$ excess energies. In addition, fcc clusters with no more than 200 atoms are observed experimentally. In this size range, both structures may thus exist.

The main difference between fcc and icosahedral structures is, of course, the order of symmetry, and the main purpose of this discussion is to emphasize its role in chemical ordering.

The simplest energetic parameter which can be considered in predicting chemical ordering is the $0 \mathrm{~K}$ binding energy of a single atom impurity as a function of position in an elemental cluster. Such a scheme was used, for instance, in Ref. 58 in order to argue why an onion structure could be expected in fcc binary clusters and not in icosahedral ones. The same calculation repeated here for the same $\mathrm{Ag}-\mathrm{Cu}$ systems as in Ref. 58, but using our EAM potential, leads to the same conclusion: $0 \mathrm{~K}$ binding energies are not consistent with the onion structure in icosahedral $\mathrm{Ag}-\mathrm{Cu}$ clusters. This conclusion thus holds using different potentials. The same argument for the $\mathrm{Cu}-\mathrm{Co}$ system, however, leads to the opposite conclusion and supports the prediction of subsurface positions of $\mathrm{Co}$ atoms in $\mathrm{Cu}$ icosahedral clusters. This is consistent with all the cases considered in the present work, even at high temperatures and above the melting point, provided the number of Co impurities is small enough (less than ten atoms, irrespective to the cluster size). It is also supported by our previous work on AgCo clusters. ${ }^{57,65}$ When a Co atom is inserted, the energy stored in relaxing $\mathrm{Ag}$ positions takes its lowest values where the coordination of $\mathrm{Ag}$ is also the lowest possible, namely, at the cluster vertices. On the other hand, the $0 \mathrm{~K}$ energy of the system is minimized both in $\mathrm{Ag}$ and in
$\mathrm{Cu}$ matrices when the coordination of Co impurity is maximized. Both conditions are met simultaneously when Co sit in the subsurface layer, beneath the vertices. This is the reason why Co substitutional impurities can be located at subsurface sites only when this is geometrically necessary. This conclusion is valid for both fcc and icosahedral structures.

As discussed in Ref. 57, this reasoning is not sufficient anymore when the number of Co atoms is larger. In this case, the $0 \mathrm{~K}$ energy gained in Co-Co binding by clustering them together is larger than the energy lost in displacing host $\mathrm{Ag}$ or $\mathrm{Cu}$ cluster atoms. The examples shown in this work mainly concern $\mathrm{Cu}-\mathrm{Co}$ clusters, but the argument pertains to $\mathrm{Ag}$-Co clusters as well. ${ }^{57}$ In the competition between lattice distortion and binding, the onion configuration may be an intermediate state triggered by surface tension, as demonstrated in Ref. 65 for CuCo fcc clusters. This was not found possible in $\mathrm{AgCo}$ clusters. ${ }^{57}$

The effect of structure is not the same here as discussed in Ref. 58. In fcc Cu-Co clusters, the Co ring is uniform. It is not in the icosahedral one, and here comes the importance of the fivefold symmetry. The fivefold symmetry configuration of small clusters is energetically more favorable than fourfold, as widely predicted computationally and observed experimentally at different temperatures. At the present stage of this discussion, we understand why a few Co impurities preferentially sit at subsurface sites beneath the 12 vertices of the icosahedron. When their number is increased above 12, the next ones will still sit at subsurface sites, displacing $\mathrm{Cu}$ atoms of low coordination and maximizing Co coordination. This latter factor, together with the energetically favorable fivefold symmetry, leads to the seven-atom subsurface decahedra found in this work. Because of the symmetry, this configuration is frustrated in fcc clusters where the trend to homogeneous onion rings is more pronounced.

The next ingredients to be considered in the problem are size and temperature. As size is concerned, the largest of the systems considered here is the easiest case. Indeed, the successive steps appear well-separated when $m$ is increasing in $\mathrm{Cu}_{561-m} \mathrm{Co}_{m}$ clusters: (i) filling subsurface vertices, (ii) forming seven-atom decahedra, (iii) filling subsurface neighboring facets, and (iv) forming a large subsurface decahedron or a central icosahedron. When the cluster size is reduced, the sequence is basically the same, but the steps are entangled because of the limited room.

The role of temperature is more subtle and probably not fully captured in this study because a direct measurement of entropy is missing. Through all the cases considered, expectedly, temperature induces disorder and, in this respect, the $0 \mathrm{~K}$ discussion above pertains as a reference. It was shown in Ref. 79 that temperature has the effect of decreasing the difference between stresses on different sublattices of an ordered alloy. If the temperature of a binary cluster is increased, one thus expects the stress field to become more uniform. This is a factor which may favor its homogenization. Different configurations have, however, different sensitivities to temperature. The seven-atom decahedral configurations, for instance, are significantly degraded, even at $300 \mathrm{~K}$. However, up to the melting point, the thermal effects are not sufficient to induce a uniform onion configuration. Temperature also monitors the statistical populations of dif- 
ferent isomeric configurations. One example may be the possible decahedral and icosahedral configurations that display very small $0 \mathrm{~K}$ configuration energy differences. The statistics of the evolution of their populations with temperature is beyond the scope of this work.

This discussion should not be closed without stressing two additional features involved in the cluster configurations. The first one is the immiscibility of the species in the bulk phase. Heats of solutions of all possible binary compounds made of $\mathrm{Ag}, \mathrm{Cu}$, and $\mathrm{Co}$ were computed positive with this EAM potential, with a maximum close to the $A_{0.5} B_{0.5}$ composition. ${ }^{65}$ In all configurations found in the present work, species tend to precipitate either in the same cluster layer or into first neighbor groups. Immiscibility thus also pertains to the icosahedral phase. In descending order of heats of solution at this composition, the classification is $\mathrm{AgCo}, \mathrm{AgCu}$, and $\mathrm{CuCo}$. The same was found in the case of clusters. This classification is consistent with the $\mathrm{Ag}$ forming the surface shell; however, it is not sufficient to describe all cases and it was shown in Ref. 65 that surface excess energy is another quantity needed to predict chemical ordering. The same methodology is used here and we show, by means of ternary clusters, that lowering the surface energy of a binary cluster by coating with a third species has profound consequences on the ordering of the inner cluster by enhancing the precipitation of Co atoms into compact groups: binding effects then dominate.

\section{CONCLUSION}

The study of immiscible icosahedral clusters with various sizes and composition presented here evidences features to which equilibrium configurations obey. First, a homogeneous mixture of two or three species immiscible in the bulk is not observed in clusters. Second, the icosahedral structure is found stable for all compositions at $10 \mathrm{~K}$ as well as at $300 \mathrm{~K}$. The third feature is the dramatic effect of a coating on the configuration of the components inside the cluster, whatever the temperature is. Finally, the importance of the icosahedral structure deserves to be emphasized. The example shown here is the Co distribution in the subsurface area. A homogeneous onionlike structure was predicted in truncated octahedral fcc CuCo clusters containing 201 atoms and more in the absence of a coating. It is found neither in the 147,309 , nor in the 561 atoms icosahedral clusters. In those, close to $0 \mathrm{~K}$, cobalt decahedra are formed based on a vertex of the subsurface shell. Such an accommodation is, of course, not favored in a cubic structure. In the icosahedral case, temperature moderates the decahedral grouping, but it also does not lead to the formation of a homogeneous onion structure.

Experimental assessment of these predictions is, in principle, possible, provided clusters may be deposited on a surface or embedded in a matrix in controlled temperature conditions. However, like in the case of a coating, interfaces may substantially modify the configurations of the different elements. These should be accounted for in the modeling.

\section{ACKNOWLEDGMENTS}

A.D. is thankful for a grant of the Fonds National de la Recherche Scientifique of Belgium in the frame of the Research Agreement No. 2.4520.03F with the Fonds de la Recherche Fondamentale Collective. I.A. is thankful for a grant of the Université Libre de Bruxelles.
*Corresponding author FAX: +32-2-6505227.

dzhurakhalov@mail.ru

${ }^{1}$ E. F. Rexer, J. Jellinek, E. B. Krissinel, E. K. Parks, and S. J. Riley, J. Chem. Phys. 117, 82 (2002).

${ }^{2}$ R. L. Johnston, T. V. Mortimer-Jones, C. Roberts, S. Darby, and F. R. Manby, Lecture Notes in Computer Science (SpringerVerlag, Berlin, 2002), Vol. 2279, pp. 25-61.

${ }^{3}$ R. L. Johnston, Dalton Trans. 22, 4193 (2003).

${ }^{4}$ D. L. Freeman and J. D. Doll, Annu. Rev. Phys. Chem. 47, 43 (1996).

${ }^{5}$ J. Urban, Cryst. Res. Technol. 33, 1009 (1998).

${ }^{6}$ R. Ferrando, J. Jellinek, and R. J. Johnston, Chem Rev. (Washington, D.C.) (to be published 2008).

${ }^{7}$ Y. Yamada and A. W. Castelman, Jr., J. Chem. Phys. 97, 4543 (1992).

${ }^{8}$ W. A. de Heer, Rev. Mod. Phys. 65, 611 (1993).

${ }^{9}$ S. Neukermans, E. Janssens, H. Tanaka, R. E. Silverans, and P. Lievens, Phys. Rev. Lett. 90, 033401 (2003).

${ }^{10}$ S. Neukermans, E. Janssens, Z. F. Chen, R. E. Silverans, P. v. R. Schleyer, and P. Lievens, Phys. Rev. Lett. 92, 163401 (2004).

${ }^{11}$ E. Janssens, S. Neukermans, X. Wang, N. Veldeman, R. E. Silverans, and P. Lievens, Eur. Phys. J. D 34, 23 (2005).

${ }^{12}$ W. Bouwen, P. Thoen, F. Vanhoutte, S. Bouckaert, F. Despa, H.
Weidele, R. E. Silverans, and P. Lievens, Rev. Sci. Instrum. 71, 54 (2000).

${ }^{13}$ D. K. Saha, K. Koga, and H. Takeo, Nanostruct. Mater. 8, 1139 (1997).

${ }^{14}$ U. Pal, J. F. Sánchez-Ramírez, H. B. Liu, A. Medina, and J. A. Ascencio, Appl. Phys. A: Mater. Sci. Process. 79, 79 (2004).

${ }^{15}$ J. L. Rodríguez-López, J. M. Montejano-Carrizales, U. Pal, J. F. Sánchez-Ramírez, H. E. Troiani, D. García, M. Miki-Yoshida, and M. José-Yacamán, Phys. Rev. Lett. 92, 196102 (2004).

${ }^{16}$ B. Pauwels, G. Van Tendeloo, E. Zhurkin, M. Hou, G. Verschoren, L. T. Kuhn, W. Bouwen, and P. Lievens, Phys. Rev. B 63, 165406 (2001).

${ }^{17}$ J. A. Ascencio, H. B. Liu, U. Pal, A. Medina, and Z. L. Wang, Microsc. Res. Tech. 69, 522 (2006).

${ }^{18}$ S. Link, Z. L. Wang, and M. A. El-Sayed, J. Phys. Chem. 103, 3529 (1999).

${ }^{19}$ K. Hiraga, T. Ohsuna, and K. Sugiyama, Rigaku J. 16, 38 (1999).

${ }^{20}$ X. Zhang, G. Li, X. Xing, X. Zhao, Z. Tang, and Z. Gao, Rapid Commun. Mass Spectrom. 15, 2399 (2001).

${ }^{21}$ A. Kolmakov and D. W. Goodman, Rev. Sci. Instrum. 74, 2444 (2003).

${ }^{22}$ C. Kan, W. Cai, C. Li, L. Zhang, and H. Hofmesiter, J. Phys. D 36, 1609 (2003). 
${ }^{23}$ D. L. Peng, T. Hihara, and K. Sumyiama, J. Magn. Magn. Mater. 277, 210 (2004).

${ }^{24}$ B. Mierzwa, J. Alloys Compd. 362, 178 (2004).

${ }^{25}$ H. Remita, J. Khatouri, M. Tréguier, J. Amblard, and J. Belloni, Z. Phys. D: At., Mol. Clusters 40, 127 (1997).

${ }^{26}$ C. Y. Tan, J. S. Chen, B. H. Liu, and G. M. Chow, J. Cryst. Growth 293, 175 (2006).

${ }^{27}$ I. Srnova-Sloufova, F. Lednicky, A. Gemplerle, and J. Gemperlove, Langmuir 16, 9928 (2000).

${ }^{28}$ S. J. Meija-Rosales, C. Fernández-Navarro, E. Pérez-Tijerina, J. M. Montejano-Carrizales, and M. José-Yacamán, J. Phys. Chem. B 110, 12884 (2006).

${ }^{29}$ A. Bol, J. A. Alonso, J. M. López, and A. Mañanes, Z. Phys. D: At., Mol. Clusters 30, 349 (1994).

${ }^{30}$ S. Wang, J. Yu, H. Mizuseki, J. Yan, Y. Kawazoe, and C. Wang, J. Chem. Phys. 120, 8463 (2004).

${ }^{31}$ S. Sahoo, G. Rollmann, and P. Entel, Phase Transitions 79, 693 (2006)

${ }^{32}$ S. Erkoç and H. Oymak, J. Phys. Chem. B 107, 12118 (2003).

${ }^{33}$ H. Oymak and S. Erkoç, Phys. Rev. A 66, 033202 (2002).

${ }^{34}$ J. A. Alonso, Phys. Scr., T 55, 177 (1994).

${ }^{35}$ P. Mitev, D. G. Papageorgiou, Ch. E. Lekka, and G. A. Evangelakis, Surf. Sci. 566-568, 937 (2004).

${ }^{36}$ S. Darby, T. V. Mortimer-Jones, R. L. Johnston, and C. Roberts, J. Chem. Phys. 116, 1536 (2002).

${ }^{37}$ N. T. Wilson and R. L. Johnston, J. Mater. Chem. 12, 2913 (2002).

${ }^{38}$ M. S. Bailey, N. T. Wilson, C. Roberts, and R. L. Johnston, Eur. Phys. J. D 25, 41 (2003).

${ }^{39}$ L. D. Lloyd, R. L. Johnston, S. Salhi, and N. T. Wilson, J. Mater. Chem. 14, 1691 (2004).

${ }^{40}$ Y. H. Chui and K. Chan, Mol. Simul. 30, 670 (2004).

${ }^{41}$ J. P. K. Doye and L. Meyer, Phys. Rev. Lett. 95, 063401 (2005).

${ }^{42}$ J. Wang, G. Wang, X. Chen, W. Lu, and J. Zhao, Phys. Rev. B 66, 014419 (2002).

${ }^{43}$ R. Ferrando, A. Fortunelli, and G. Rossi, Phys. Rev. B 72, 085449 (2005).

${ }^{44}$ Q. L. Lu, L. Z. Zhu, L. Ma, and G. H. Wang, Chem. Phys. Lett. 407, 176 (2005).

${ }^{45}$ Q. L. Lu, L. Z. Zhu, L. Ma, and G. H. Wang, Phys. Lett. A 350, 258 (2006).

${ }^{46}$ G. Rossi, R. Ferrando, A. Rapallo, A. Fortunelli, B. C. Curley, L. D. Lloyd, and R. L. Johnston, J. Chem. Phys. 122, 194309 (2005).

${ }^{47}$ J. L. Rodríguez-López, J. M. Montejano-Carrizales, and M. JoséYacamán, Appl. Surf. Sci. 219, 56 (2003).

${ }^{48}$ S. C. Hendy and B. D. Hall, Phys. Rev. B 64, 085425 (2001).

${ }^{49}$ D. Cheng, S. Huang, and W. Wang, Eur. Phys. J. D 39, 41 (2006).

${ }^{50}$ F. Leroy, G. Renaud, A. Letoublon, R. Lazzari, C. Mottet, and J.
Goniakowski, Phys. Rev. Lett. 95, 185501 (2005).

${ }^{51}$ C. Mottet, G. Rossi, F. Baletto, and R. Ferrando, Phys. Rev. Lett. 95, 035501 (2005).

${ }^{52}$ P. Müller and C. Mottet, J. Comput. Theor. Nanosci. 4, 316 (2007).

${ }^{53}$ V. Moreno, J. Creuze, F. Berthier, C. Mottet, G. Treglia, and B. Legrand, Surf. Sci. 600, 5011 (2006).

${ }^{54}$ E. E. Zhurkin and M. Hou, J. Phys.: Condens. Matter 12, 6735 (2000).

${ }^{55}$ T. Van Hoof and M. Hou, Appl. Surf. Sci. 226, 94 (2004).

${ }^{56}$ T. Van Hoof and M. Hou, Eur. Phys. J. D 29, 33 (2004).

${ }^{57}$ T. Van Hoof and M. Hou, Phys. Rev. B 72, 115434 (2005).

${ }^{58}$ F. Baletto, C. Mottet, and R. Ferrando, Phys. Rev. Lett. 90, 135504 (2003).

${ }^{59}$ L. Yang, J. Z. Jiang, T. Liu, T. D. Hu, and T. Uruga, Appl. Phys. Lett. 87, 061918 (2005).

${ }^{60}$ J. A. Barrow, D. J. Sordelet, M. F. Besser, C. J. Jenks, P. A. Thiel, E. F. Rexer, and S. J. Riley, J. Phys. Chem. A 106, 9204 (2002).

${ }^{61}$ M. Polak and L. Rubinovich, Surf. Sci. 584, 41 (2005).

${ }^{62}$ L. Rubinovich and M. Polak, Phys. Rev. B 69, 155405 (2004).

${ }^{63}$ M. Polak and L. Rubinovich, Phys. Rev. B 71, 125426 (2005).

${ }^{64}$ L. Rubinovich, M. I. Haftel, N. Bernstein, and M. Polak, Phys. Rev. B 74, 035405 (2006).

${ }^{65}$ A. A. Dzhurakhalov and M. Hou, Phys. Rev. B 76, 045429 (2007).

${ }^{66}$ M. S. Daw and M. I. Baskes, Phys. Rev. B 29, 6443 (1984).

${ }^{67}$ R. A. Johnson, Phys. Rev. B 39, 12554 (1989).

${ }^{68}$ R. A. Johnson, Phys. Rev. B 41, 9717 (1990).

${ }^{69}$ M. Hou, M. El Azzaoui, H. Pattyn, J. Verheyden, G. Koops, and G. Zhang, Phys. Rev. B 62, 5117 (2000).

${ }^{70}$ R. Najafabadi, D. J. Srolowitz, E. Ma, and M. Atzmon, J. Appl. Phys. 74, 3144 (1993).

${ }^{71}$ T. Klassen, U. Herr, and R. S. Averback, Acta Mater. 45, 2921 (1997).

${ }^{72}$ J. L. Murray, Metall. Trans. A 15A, 261 (1984).

${ }^{73}$ G. Mazzone, V. Rosato, M. Pintore, F. Delogu, P. F. Demontis, and G. B. Suffritti, Phys. Rev. B 55, 837 (1997).

${ }^{74}$ H. W. Sheng and E. Ma, Phys. Rev. B 61, 9979 (2000).

${ }^{75}$ C. Gente, M. Oehring, and R. Bormann, Phys. Rev. B 48, 13244 (1993).

${ }^{76}$ M. Haebe and T. Nishizawa, CALPHAD: Comput. Coupling Phase Diagrams Thermochem. 4, 83 (1980).

${ }^{77}$ R. L. Johnston, Atomic and Molecular Clusters (Taylor \& Francis, London, 2002).

${ }^{78}$ A. Sebecti and Z. B. Guvenc, Modell. Simul. Mater. Sci. Eng. 12, 1131 (2004).

${ }^{79}$ E. E. Zhurkin, G. Hautier, and M. Hou, Phys. Rev. B 73, 094108 (2006). 